\section{Case Report}

Korean J Transplant 2021;35:200-206 https://doi.org/10.4285/kjt.21.0005
Received April 2, 2021

Revised August 5, 2021

Accepted August 30, 2021

Corresponding author: Byunghyun Choi Department of Surgery, Pusan National University Yangsan Hospital, 20 Geumoro, Mulgeum-eup, Yangsan 50612, Korea Tel: +82-55-360-2478

Fax: +82-55-360-2154

E-mail: gmoolpop@gmail.com

(C) The Korean Society for Transplantation This is an Open Access article distributed under the terms of the Creative Commons Attribution Non-Commercial License (http://creativecommons.org/licenses/ by-nc/4.0/) which permits unrestricted non-commercial use, distribution, and reproduction in any medium, provided the original work is properly cited.

\section{$\mathrm{K} \mathrm{T}^{\mathrm{K}}$ \\ KOREAN JOURNAL OF TRANSPLANTATION}

pISSN 2671-8790

elSSN 2671-8804

\title{
Reusing hepatic grafts in Korea: a case report
}

\author{
Hye Young Kim ${ }^{1}$, Byunghyun $\mathrm{Choi}^{2}$, Minhwa Kim${ }^{1}$, YounJung Choi ${ }^{1}$, Jeongrim Lee ${ }^{1}$, \\ Won Hyun $\mathrm{Cho}^{1}$ \\ ${ }^{1}$ Korea Organ Donation Agency, Seoul, Korea \\ ${ }^{2}$ Department of Surgery, Pusan National University Yangsan Hospital, Pusan National University School of \\ Medicine, Yangsan, Korea
}

The shortage of donor organs has compelled transplant centers to use organs from non-standard donors. The Korean Network for Organ Sharing data showed that there were 5,804 potential recipients on the waiting list, and only 1,579 patients underwent liver transplant in 2019. Reuse of a graft that has been transplanted previously to other recipients could be an option in this situation. However, given the susceptibility of hepatic grafts to ischemic damage, their reuse must be considered extremely carefully. In this retrospective, observational study, we investigated the outcomes of six cases of hepatic graft reuse in Korea since the year 2000, from information gathered from patient medical records from ten transplant centers. Only three of the six reused hepatic grafts functioned well. Among the three successful transplants, two had minimal ischemic damage owing to a longer interval between the first and second transplants, and because they were obtained from living donors. Two of the five cadaveric transplants were successful. The outcome of reusing hepatic grafts in Korea has not been ideal. However, in patients with limited choices, it can be carefully considered, provided the graft is thoroughly checked for ischemic damage and the recipient status is ascertained.

Keywords: Organ transplantation; Hepatic transplantation; Graft reuse; Graft survival; Case report

\section{INTRODUCTION}

The number of cadaveric organ donors has markedly increased since the revision of the "Internal organs, etc. transplant act" in 2011. However, the gap between the number of patients on the waiting list and the number of donors has only grown wider. In the year 2019, the number of patients on the waiting list for organ transplantation was 32,560 , while the number of transplants performed was only 1,612 from 450 cadaveric donors. According to Korean Network for Organ Sharing data, that there were 5,804 potential recipients on the waiting list; however, only 1,579 patients underwent liver transplant in 2019 [1].
This shortage of organs has compelled transplant centers to use organs from non-standard donors. Reuse of a graft that has been previously transplanted to other recipients can be a viable option in this situation. However, because the liver is generally more susceptible to ischemic damage than the kidney, reusing hepatic grafts without careful consideration can be dangerous [2,3].

Reports on hepatic graft reuse have been published in other countries; however, with the exception of two case reports, there are no national reports on the same in Korea [4-7]. Since the year 2000, there have been 24 cases of graft reuse across all transplant centers in Korea, wherein grafts were sourced from brain-dead organ donors who 


\section{HIGHLIGHTS}

- There is a severe shortage of donors for organ transplantation.

- Grafts can be reused to address this shortage.

- Hepatic grafts are susceptible to ischemic damage; reuse requires careful evaluation.

had previously received the same graft themselves. Among them, we focused on six transplants that reused a hepatic graft. All data were collected retrospectively through electronic medical records after obtaining permission from the Institutional Review Board (IRB) of each transplant center (Seoul National University Bundang Hospital: No. B-2004604-110, Pusan National University Yangsan Hospital: No. 05-2020-045, Asan Medical Center: No. S2021-0010-0001, The Catholic University of Korea, Seoul St. Mary's Hospital: No. KC21RIDI0146). Informed consent was waived given the retrospective design of the study. Transplant surgeons who plan to reuse hepatic grafts for their patients may benefit from our nation-wide data.

\section{CASE REPORT}

Six recipients underwent liver transplantation with reused hepatic grafts; one underwent living-donor transplantation, while the others underwent deceased-donor transplantation. Data on the warm ischemic time were unavailable for all patients; therefore, we have only shown the cold ischemic times of the first and second transplantations (Table 1). The model for end-stage liver disease (MELD) scores in the failed graft groups were higher than those in the successful graft groups, even though the absolute values were not high.

\section{Case 1}

The first recipient was a 32-year-old woman with fulminant hepatitis $A$. The donor was a 30-year-old woman, the younger sister of the recipient. A modified right lobe graft was used for living donor transplantation. However, the next day, the recipient developed brain edema as observed on a computed tomography (CT) scan, and brain death occurred after one day. Even though the abrupt huge tragedy occurred to the family, they agreed to donation of the first recipient as a deceased donor.

Eight days after the first transplant procedure, the graft was re-transplanted to a 43-year-old man with liver cirrhosis secondary to hepatitis $B$ virus (HBV) infection. He was on the waiting list for deceased donor liver transplantation for intractable ascites. There were mild adhesions around the liver graft, and mobilization of the liver was uneventful. The bile duct, portal vein, hepatic artery, and reconstructed hepatic vein were clearly identified and procured from the former anastomotic site and confirmed to be in a good condition for subsequent anastomosis. The graft weight had increased from $590 \mathrm{~g}$ at the first transplantation to $1,120 \mathrm{~g}$ at the second transplantation. The graft-recipient weight ratio was $1.66 \%$. He has been doing well for more than 10 years after the transplant. This case has been previously reported [5] (Fig. 1A).

\section{Case 2}

The first recipient was a 42-year-old man with toxic hepatitis. The donor was a 37-year-old man who had suffered brain death due to subarachnoid hemorrhage (SAH). However, hepatic encephalopathy developed after the transplant, and brain necrosis was observed on a CT scan seven days after the transplant.

The graft was then retransplanted to a 45-year-old man with liver cirrhosis and variceal bleeding due to HBV infection. A duct-to-duct anastomosis was performed and an internal stent was inserted; no problems were noted in the duct anastomosis during the graft-reuse surgery. Furthermore, there were no major problems, such as thinning of the portal vein on the graft side; therefore, there was no risk of bleeding. The anastomosis was created using the branch-patch technique, which involved the gastroduodenal and common hepatic arteries; no additional vessel was obtained from the donor during the anastomosis. A piggyback cavocaval anastomosis was performed; unlike in the original transplantation, the anastomosis was excised proximally. The graft function did not recover after the second transplant. The recipient then underwent transplant from a different brain-dead donor three days after the earlier transplant with the reused hepatic graft (Fig. 2A).

\section{Case 3}

The first recipient was a 48-year-old woman with idiopathic hepatic failure and hepatic encephalopathy. The donor was a 31-year-old woman who had been declared brain dead due to SAH. However, the recipient's encephalopathy did not recover despite the transplant. The patient deteri- 


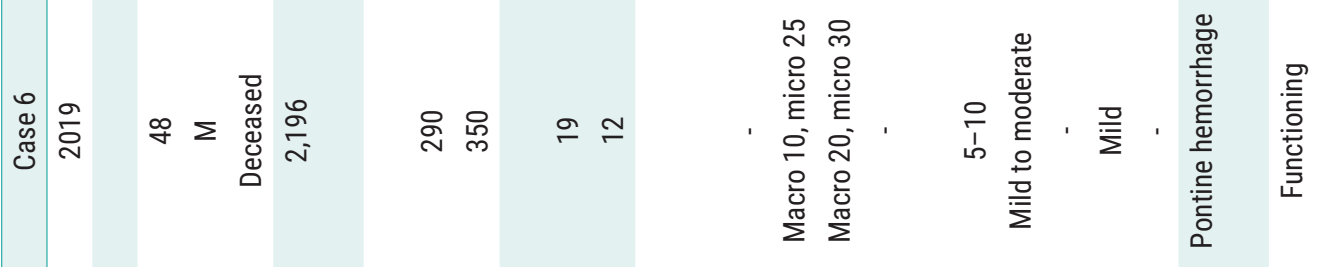

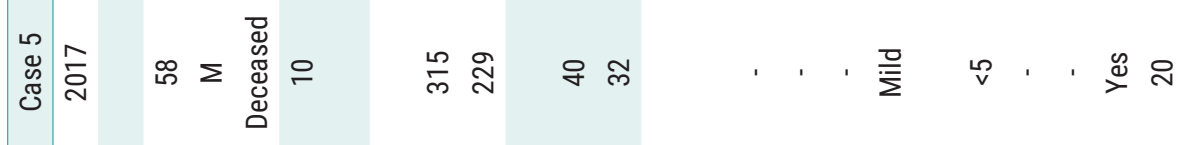

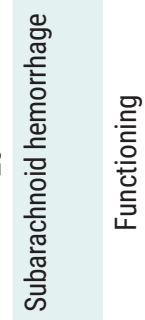

离

బ

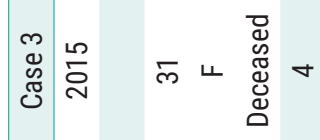

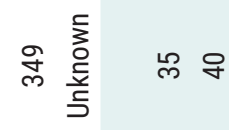

$\frac{\stackrel{0}{\frac{\pi}{\pi}}}{\frac{\pi}{\frac{\pi}{0}}}$

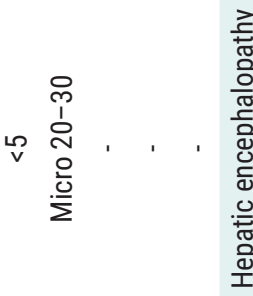

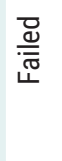

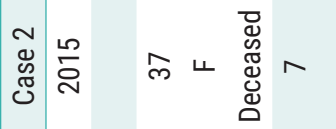

$\stackrel{\text { ๙ }}{\sim} \underset{\sim}{\sim}$

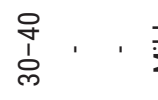

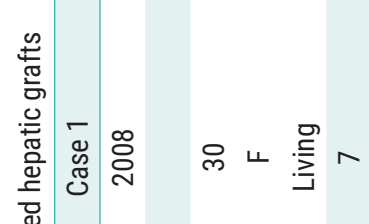

\& $6 \quad \infty$

을

ᄂ

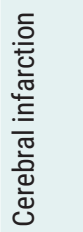

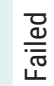

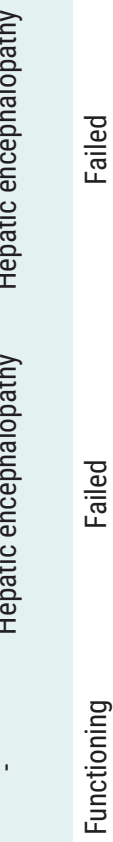

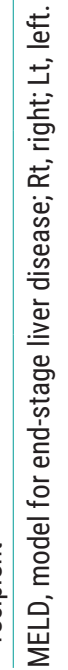



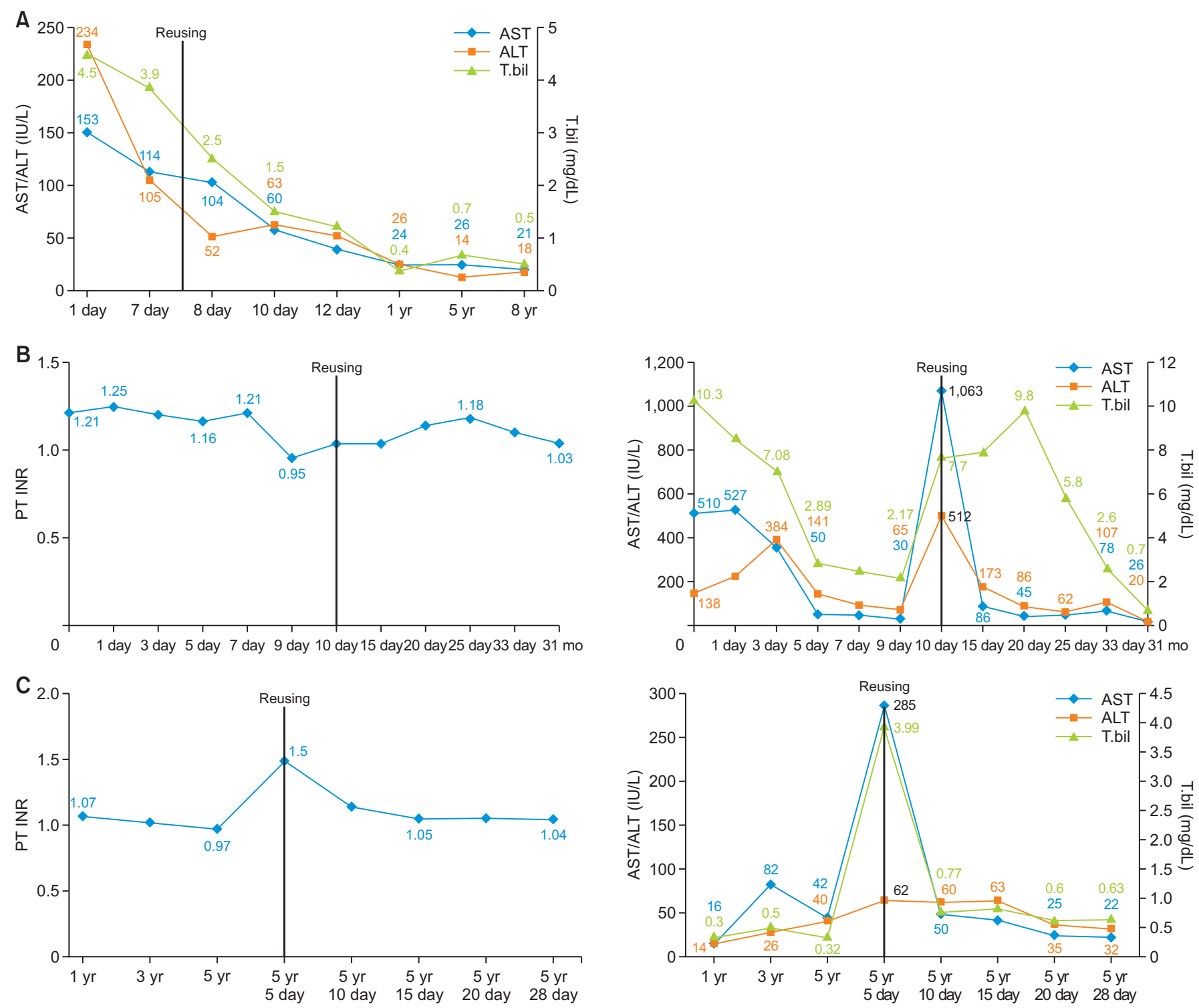

Fig. 1. Change in biochemical parameters of the first and second recipient in case 1 (A), case 5 (B), case 6 (C, successful group). AST, aspartate aminotransferase; ALT, alanine aminotransferase; PT, prothrombin time; INR, international normalized ratio; T.bil, total bilirubin.

orated and was ultimately declared brain dead three days after transplant.

The second recipient was a patient with alcoholic liver cirrhosis. Hepatic vein anastomosis was performed using the piggyback technique, and the donor hepatic artery was easily anastomosed with the recipient common hepatic artery. An end-to-end bile duct anastomosis was performed as well. However, postoperative bleeding occurred and necrosis of segments 6 and 7 was observed during re-exploration. The reused hepatic graft did not recover its function after transplant. The recipient died on the 6th day posttransplant due to primary non-function and septic shock (Fig. 2B).

\section{Case 4}

The first recipient was a 37-year-old man with toxic hepatitis secondary to HAV. The donor was a 55-year-old man who had been brain dead due to intracranial hemorrhage. After the transplant, brain infarction and edema developed, ending in brain death 11 days after transplant.

The second recipient was a 53-year-old man with HBV and liver cirrhosis. In case of the first recipient, hepatecto- 

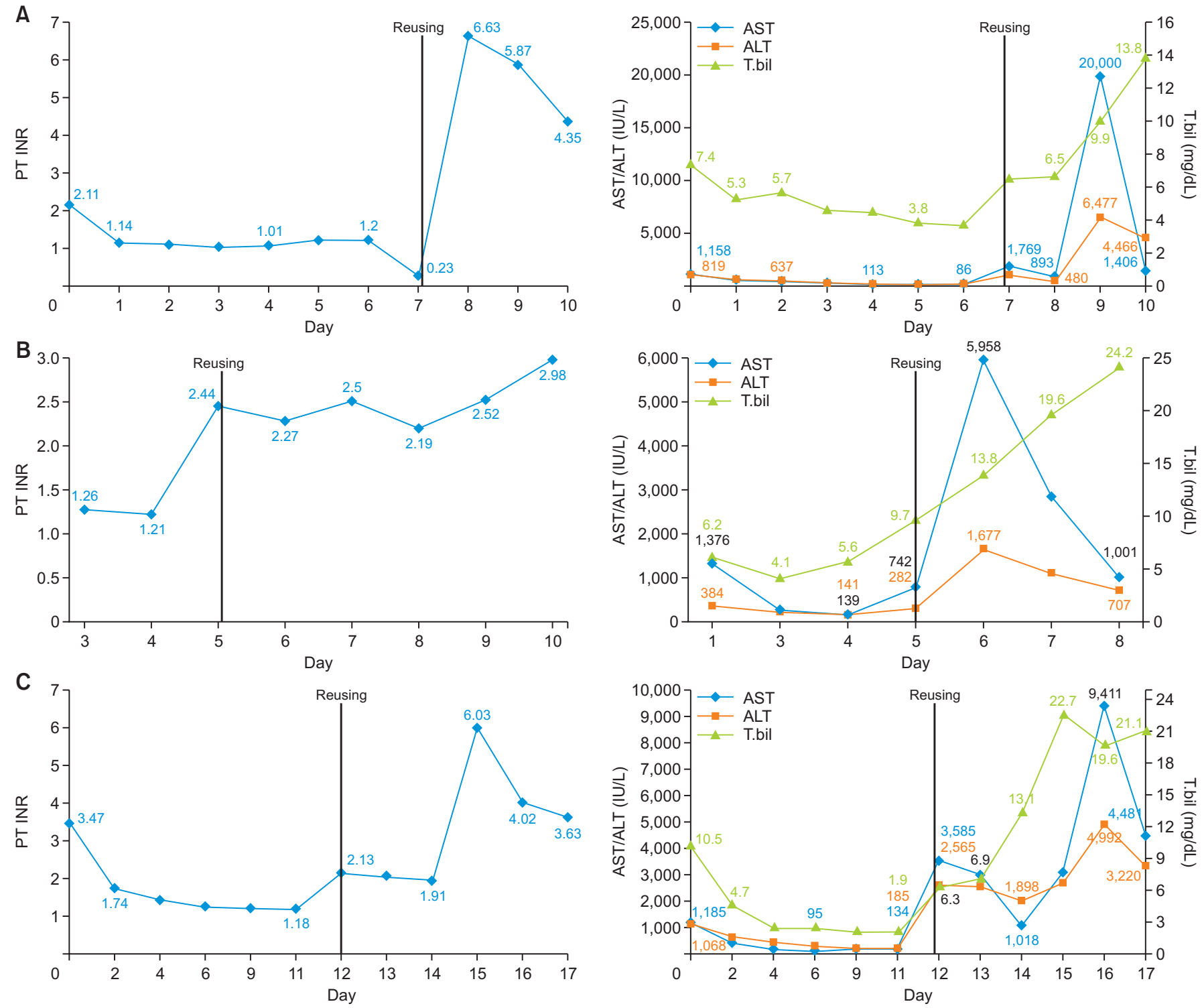

Fig. 2. Change in biochemical parameters of the first and second recipient in case 2 (A), case $3(B)$, and case 4 (C, the unsuccessful group). PT, prothrombin time; INR, international normalized ratio; AST, aspartate aminotransferase; ALT, alanine aminotransferase; T. bil, total bilirubin.

my was performed in the usual manner without any difficulties. The second recipient had already undergone living donor liver transplantation (LDLT) 4 years ago. Therefore, the individual dissection of the hepatic hilum was very difficult due to adhesion. Furthermore, the patient had a cavernous malformation of the portal vein before the LDLT, and a portal vein stent had been inserted after the LDLT. Therefore, the portal vein flow was very weak, and the surgeon anastomosed the left renal vein to the graft portal vein by using the cadaveric iliac vein (reno-portal anastomosis). Postoperative CT and Doppler ultrasound revealed a decreased flow in the hepatic artery and portal vein. and the reused hepatic graft did not recover its function after transplant, and the recipient died on the 7th day posttransplant due to primary non-function and septic shock (Fig. 2C).

\section{Case 5}

The first recipient was a 42-year-old man with alcoholic liver cirrhosis undergoing hemodialysis for end-stage kidney disease. The donor was a 58-year-old man who had been brain dead due to SAH. On the 8th day after transplant, subdural hemorrhage occurred and the recipient experi- 
enced brain death.

The second recipient was a 40-year-old woman with hepatic failure caused by toxic hepatitis. Because the liver graft was reconstructed by using the piggyback technique in the first recipient, the recipient retrohepatic inferior vena cava (IVC) was completely preserved for application of the modified piggyback technique. The weight of the liver graft was $1,430 \mathrm{~g}$. The redundant portion of the hepatic artery, which was derived from the first recipient, was resected and the graft's own hepatic artery was anastomosed to the hepatic artery stump of the second recipient. Biliary reconstruction was performed in duct-to-duct anastomosis of the common bile duct with a T-tube insertion. The recipient recovered well and has been doing well since. This case also has been reported previously (Fig. 1B) [4].

\section{Case 6}

The first recipient was a 65 -year-old man with hepatitis $\mathrm{C}$, liver cirrhosis, and alcoholic liver disease. The donor was a 48-year-old man with SAH. The recipient recovered well after transplant. However, 6 years later, pontine hemorrhage occurred, abruptly leading to brain death. The second recipient, who had underlying hepatocellular carcinoma as the indication for transplant. No complicated procedures, except for adhesiolysis, were performed during the transplantation. Duct-to-duct anastomosis was performed in an end-to-end manner. The surgeon used the retrohepatic IVC, which included the liver graft, and performed hepatic vein anastomosis with the piggyback cavocaval technique. The recipient recovered well and has been doing well since the procedure (Fig. 1C).

\section{DISCUSSION}

Reusing organs in an organ shortage can be a good option in terms of increasing the donor pool. However, the reuse of a liver graft can be risky for patients, unlike reuse of the kidney [3]. In this study, the first case received a graft that was relatively less ischemic due to living donor transplant; in the last case, there was virtually no ischemic damage because the second transplant occurred 6 years after the first. Except for the last case, one of the remaining four livers from deceased donors was liver transplanted successfully.

In a recent study of reused hepatic grafts, there was a proposal to consider routine biopsy before the second transplant to predict primary non-functional graft status
[8]. However, according to our study, non-functional status seems quite non-predictable (cases 2 and 3). Laboratory findings are likely more important than biopsy findings. If the total bilirubin level is $3 \mathrm{mg} / \mathrm{dL}$ or higher in the donor, the graft can be considered unsuitable for transplant. Since June 2016, the distribution of liver grafts has changed according to the MELD system; a higher MELD score reflects poorer patient condition before transplant, meaning the hepatic graft would experience a greater burden despite the transplant. In such situations, reusing the hepatic graft would necessitate more caution.

Hepatic ischemia-reperfusion injury (IRI) is a major risk factor of liver transplant failure [2]. In cases 2 and 3, the reused graft experienced two consecutive IRIs during a relatively short period. Furthermore, in case 3 , the MELD score of the second recipient was very high (40) and the international normalized ratio and total bilirubin level had already increased in the first recipient before the second transplant.

In case 4 , the recipient had a cavernous malformation of the portal vein and the portal vein stent had already been inserted before the second transplant. Unfortunately, reno-portal bypass was unsuccessful. In this case, the graft condition might have not been the primary cause of graft failure; careful recipient selection appears to be crucial in the transplantation of reused grafts.

This study has some limitations. We could not obtain data of good quality due to the study's retrospective and multi-center design. The data were somewhat old and had different available ranges according to the IRB policies of the respective institutions. However, we hope that our report helps other liver transplant surgeons who are planning to reuse hepatic grafts. In conclusion, to date, the outcome of reusing hepatic grafts in Korea has not been ideal. However, in patients with limited options, reuse can be carefully considered after thoroughly assessing the graft for ischemic damage and status of the recipient.

\section{ACKNOWLEDGMENTS}

\section{Conflict of Interest}

No potential conflict of interest relevant to this article was reported.

\section{Funding/Support}

This study was supported by research grant from the Kore- 
an Society for Transplantation (2021-00-03008-020).

\section{ORCID}

Hye young Kim

https://orcid.org/0000-0002-1776-3798

Byunghyun Choi

Minhwa Kim

YounJung Choi

Jeongrim Lee

Won Hyun Cho

https://orcid.org/0000-0002-9245-5066

https://orcid.org/0000-0003-1879-1499

https://orcid.org/0000-0003-4542-8169

https://orcid.org/0000-0001-6038-5115

https://orcid.org/0000-0001-5916-6601

\section{Author Contributions}

Conceptualization: HYK . Data curation: MK, YJC. Formal analysis: HYK, BHC. Methodology: HYK, MK, YJC. Supervision: JL, WHC. Investigation: MK, YJC. Writing-original draft: HYK, BHC. Writing-review \& editing: JL, WHC.

\section{Additional Contributions}

The authors wish to thank several hospitals for providing IRB approval and accurate data.

\section{REFERENCES}

1. Korean Network for Organ Sharing (KONOS). KONOS waiting list [Internet]. Seoul: KONOS; 2020 [cited 2021 Sep 1]. Available from: https://www.konos.go.kr.
2. Rampes $S, M a D$. Hepatic ischemia-reperfusion injury in liver transplant setting: mechanisms and protective strategies. J Biomed Res 2019;33:221-34.

3. Woo EJ, Kim M, Choi YJ, Lee J, Cho WH, Park Cl, et al. Outcomes of reused transplanted kidneys using cases from the Korean Network for Organ Sharing database. Korean J Transplant 2021;35:112-5.

4. Kim MJ, Hwang S, Jung DH, Park GC, Song GW, Cho $\mathrm{HD}$, et al. Reuse of liver allograft from a brain-dead recipient: a case report. Ann Hepatobiliary Pancreat Surg 2020;24:192-7.

5. Hu XG, Kim IG, Wang HJ, Kim BW, Hong SY, Kim YB, et al. Reuse of living-donor liver graft in second recipient with long-term survival. Transplant Proc 2018;50:39847.

6. Ortiz J, Reich DJ, Manzarbeitia C, Humar A. Successful re-use of liver allografts: three case reports and a review of the UNOS database. Am J Transplant 2005;5:189-92.

7. Rubay R, Wittebolle X, Ciccarelli O, Roggen F, Talpe S, Laterre PF, et al. Re-use of a liver allograft; an exceptional opportunity to enlarge the organ donor pool. Transpl Int 2003;16:497-9.

8. Tanaka H, McAlister VC, Levstik MA, Ghent CN, Marotta PJ, Quan D, et al. Reuse of liver grafts following the brain death of the initial recipient. World $\mathrm{J}$ Hepatol 2014;6:443-7. 